\title{
Integrated transcriptomic and epigenetic data analysis identifiesaberrant expression of genes in acute myeloid leukemia with MLL-AF9 translocation
}

\author{
FANGCE WANG $^{1}$, ZHENG LI $^{1}$, GUANGMING WANG ${ }^{1}$, XIAOXUE TIAN ${ }^{1}$, JIE ZHOU $^{1}$, WENLEI YU ${ }^{1}$, \\ ZHUOYI FAN $^{1}$, LIN DONG ${ }^{1}$, JINYUAN LU ${ }^{1}$, JUN XU $^{2}$, WENJUN ZHANG ${ }^{1}$ and AIBIN LIANG ${ }^{1}$ \\ ${ }^{1}$ Department of Hematology, Tongji Hospital, Tongji University School of Medicine, Shanghai 200092; \\ ${ }^{2}$ Medical Center for Stem Cell Engineering and Transformation, East Hospital, \\ Tongji University School of Medicine, Shanghai 200120, P.R. China
}

Received June 19, 2018; Accepted December 14, 2018

DOI: $10.3892 / \mathrm{mmr} .2019 .10849$

\begin{abstract}
Rearrangement of the mixed lineage leukemia (MLL; also known as lysine methyltransferase $2 A$ ) gene is a recurrent genomic aberration in acute myeloid leukemia (AML). MLLT3, super elongation complex subunit (AF9) is one of the most common MLL fusion partners in AML. The present study aimed to explore the aberrant expression of genes associated with the MLL-AF9 translocation and identified potential new targets for the therapy of AML with MLL-AF9 translocation. The transcriptomic and epigenetic datasets were downloaded from National Center of Biotechnology Information Gene Expression Omnibus (GEO) database. Differentially expressed genes were obtained from two independent datasets (GSE68643 and GSE73457). Gene Ontology biological process and Kyoto Encyclopedia of Genes and Genomes pathway enrichment analysis was performed using the Database for Annotation, Visualization and Integrated Discovery. MLL-AF9-associated chromatin immunoprecipitation sequencing (ChIP-Seq) data was analyzed and identified binding sites for MLL-AF9 and wild type MLL (MLL WT). The ChIP-Seq of histone modification data was downloaded from the GEO database, including histone 3 lysine 4 trimethylation (H3K4me3), histone 3 lysine 79 dimethylation (H3K79me2) and histone 3 lysine 27 acetylation (H3K27ac), was used for comparing histone modification marks between the MLL-AF9 leukemia cells and normal hematopoietic cells
\end{abstract}

Correspondence to: Dr Wenjun Zhang or Dr Aibin Liang, Department of Hematology, Tongji Hospital, Tongji University School of Medicine, 1,239 Siping Road, Shanghai 200092, P.R. China E-mail: zhangwenjun@tongji.edu.cn

E-mail: lab7182@tongji.edu.cn

*Contributed equally

Key words: MLL-AF9 translocation, transcriptomic and epigenetic abnormalities, bioinformatics at MLL-AF9 and MLL WT binding sites. The differentially expressed genes with the same trend in H3K79me2, H3K27ac and $\mathrm{H} 3 \mathrm{~K} 4 \mathrm{me} 3$ alteration were identified as potential MLL-AF9 direct target genes. Upon validation using RNA-Seq data from the Therapeutically Applicable Research to Generate Effective Treatments AML project, eight potential direct target genes of MLL-AF9 were identified and further confirmed in MLL-AF9 mouse model using reverse transcription-quantitative polymerase chain reaction. These genes may have a critical role in AML with MLL-AF9 translocation.

\section{Introduction}

Recurrent genomic aberrations are common in acute myeloid leukemia (AML). The mixed lineage leukemia ( $M L L)$ gene (also known as lysine methyltransferase $2 A$ ) is a histone 3 lysine 4 (H3K4) methyltransferase involved in hematopoiesis. Rearrangement of the $M L L$ gene is present in 5-10\% of AML cases in adults and in 35-50\% of infant patients with $\operatorname{AML}(1,2)$. Patients with $M L L$ rearrangements are generally classified as a high-risk group by the World Health Organization (3). The N-terminus of the $M L L$ gene is fused to $>60$ different genes via chromosome translocations in cancer, and the most common fusion partner is MLLT3, super elongation complex subunit (AF9) (4). Leukemia with MLL-rearrangements has lower frequency of somatic mutations compared with other cancer types, as revealed by genome-scale sequencing (5). However, deregulated chromatin signatures and gene transcription are characteristic of leukemia cases exhibiting MLL-rearrangements.

A previous study demonstrated that MLL fusion proteins recruit DOT1 like histone lysine methyltransferase (DOT1L) to target loci resulting in elevated levels of histone $\mathrm{H} 3$ dimethylation at lysine 79 (H3K79me2) (6). Another study revealed that specific MLL-AF9 target genes were all marked by H3K79me2, histone H3 acetylation at lysine 27 (H3K27ac) and histone $\mathrm{H} 3$ trimethylation at lysine 4 (H3K4me3) (7). These studies performed chromatin immunoprecipitation (ChIP) to identify the aberrant pattern of chromatin signatures in leukemia cells with MLL-rearrangements; however, this 
was not compared with normal hematopoietic cells. Ji et al (8) proposed the use of differential principal component analysis (dPCA) for efficiently analyzing differential chromatin signatures from large amounts of ChIP-sequencing (Seq) data between two biological conditions.

The present study aimed to identify differentially expressed genes (DEGs) and differential chromatin signatures in MLL-AF9 binding sites between MLL-AF9 leukemia cells and normal hematopoietic cells via bioinformatic methods. The association of differential chromatin signatures with DEGs was assessed to obtain a better understanding of deregulated transcriptome and epigenome in MLL-AF9 leukemia cells. The current study provided new potential targets for AML therapy.

\section{Materials and methods}

Acquisition of gene expression datasets and ChIP-Seq data. The National Center of Biotechnology Information (NCBI) Gene Expression Omnibus (GEO) database (ncbi.nlm.nih. gov/geo) was searched and microarray expression data (GSE68643) and RNA-Seq data (GSE73457) were downloaded for comparing MLL-AF9 retrovirus infected and wild-type (WT) mouse bone marrow cells. Unprocessed data sets for microarray (.cel files) and RNA-Seq (.sra files) were used for further analysis.

DEG analysis. DEGs were identified using GEO2R (ncbi.nlm.nih.gov/geo/geo2r/) from a microarray dataset (GSE68643). The probe was annotated as an official gene symbol by the corresponding annotation files (GPL16570). For RNA-Seq data (GSE73457), sra files were converted into the fastq format using the SRA Toolkit version 2.9.1 (ncbi.nlm.nih.gov/Traces/sra/?view=software). Reads were aligned to the mouse Ensemble (GRCm38.p6) reference genome using HISAT2 version 2.0.4 (ccb.jhu. edu/software/hisat2/index.shtml) (9). Aligned reads were counted using HTSeq version 0.5.4p3 (htseq.readthedocs. io/en/release_0.10.0/) and summarized at the gene level guided by the gene annotation file in GTF format from Ensembl (ftp://ftp.ensembl.org/pub/release-94/gtf/mus_musculus) (10). The expression levels of genes was calculated using cufflinks version 2.2.0 (cole-trapnell-lab.github.io/cufflinks/) and normalized to reads per kilobase per million (RPKM) (11). DESeq2version 1.18.1 (bioconductor.org/packages/release/ bioc/html/DESeq2.html) was applied to analyze the differential expression of genes (12). Only those genes with a $\log _{2}$ fold change $(\mathrm{FC})>1$ and Benjamini and Hochberg adjusted $\mathrm{P}<0.05$ were recognized as significantly differentially expressed in microarray and RNA-Seq data. Pearson's correlation coefficient of $\log 2 \mathrm{FC}$ was used to measure common DEGs reliability between two expression datasets (GSE68643 and GSE73457).

Gene Ontology (GO) and Kyoto Encyclopedia of Genes and Genomes (KEGG) pathway analysis. The Database for Annotation, Visualization and Integrated Discovery (DAVID) database was used to detect GO categories and KEGG pathways with significant over-representation in DEGs compared with the whole genome (13). The significantly enriched biological processes and KEGG were identified as Benjamini-Hochberg adjusted $\mathrm{P}<0.05$.
Identification of MLL-AF9 and WT MLL binding sites in MLL-AF9 leukemia. To identify the binding sites of MLL-AF9 and WT MLL, ChIP-Seq data obtained using antibodies against the N-terminus of MLL-1 and the C-terminus of AF9 in various GEO datasets (GSE89336, GSE79899, GSE54344 and GSE83671) were downloaded. Model-based Meta-analysis of ChIP (MM-ChIP) software (http://liulab.dfci.harvard. edu/MM-ChIP/MMChIP-1.0.tar.gz) was applied in the cross-study integrative analysis of MLL and AF9 ChIP-Seq data (14). The MLL and AF9 binding peaks were overlapped to identify considerable MLL-AF9-binding peaks using the mergePeaks function from Hypergeometric Optimization of Motif EnRichment (HOMER) version 4.8 (homer.ucsd. edu/homer/) software (15). MLL binding peaks without AF9 signal were defined as MLL WT binding sites.

Analysis of differential chromatin patterns at the WT MLL and MLL-AF9 binding sites. The ChIP-Seq data of histone modifications downloaded from the GEO database are summarized in Table I. Using dPCA (www.biostat.jhsph.edu/dpca), the present study analyzed differential levels of $\mathrm{H} 3 \mathrm{~K} 4 \mathrm{me} 3$, H3K27ac and H3K79me2 at WT MLL and MLL-AF9 binding sites in MLL-AF9 leukemia cells and normal hematopoietic cells. Differential principal components (dPCs) with high signal-to-noise ratio (SNR) were considered reliable dPCs to report. The cut-off SNR value $(\mathrm{SNR}>5)$ was based on a previous study (8). dPCA calculated the false discovery rate (FDR) and $\log _{2}$ FC of the ChIP-Seq binding signal of each dPC. Differential sites of reliable dPCs were defined at a 5\% FDR level. Genome regions with differential ChIP-Seq binding signals were annotated by HOMER software.

Validation of potential direct targets of MLL-AF9 genes. The gene expression data were downloaded from the UCSC genome browser database (https://xenabrowser.net/ datapages/?cohort=TCGA\%20TARGET\%20GTEx\&remove $\mathrm{Hub}=$ https $\% 3 \mathrm{~A} \% 2 \mathrm{~F} \% 2 \mathrm{Fxena}$.treehouse.gi.ucsc.edu\%3A443), which contained RNA-seq data from three large cohorts, such as TCGA (https://portal.gdc.cancer.gov/), TARGET (https://ocg. cancer.gov/programs/target) and GETx (https://commonfund. nih.gov/GTEx/).

Differential expression between AML samples with MLL-AF9 translocation and normal blood samples was performed using DESeq2 version 1.18.1 (bioconductor. org/packages/release/bioc/html/DESeq2.html). All of the input parameters were default values.

Generation of MLL-AF9 leukemia models. Bone marrow transplantation studies were performed as previously described (16). Briefly, C57BL/6 female mice (12 week old; Shanghai SLAC laboratory Animal Co., Ltd.) were used as donors and recipients. A total of 22 recipients mice were housed in specific pathogen-free (SPF) conditions under a $12 \mathrm{~h}$ light-dark cycle at $22 \pm 1^{\circ} \mathrm{C}$, humidity of $50-60 \%$ with free access to food and water. The mice were randomly divided into control and MLL-AF9 model groups $(n=11$ in each group; weight, $\sim 20 \mathrm{~g}$ ). The donor mice were sacrificed and lineage negative hematopoietic progenitors were obtained from femurs and tibias using Lineage Cell Depletion mouse kit and LS MACS columns (Miltenyi Biotec, Inc.) and 
Table I. Chromatin immunoprecipitation-Seq data of histone modifications downloaded from GEO database.

\begin{tabular}{|c|c|c|}
\hline GEO Sample ID & Cell type & Experiment type \\
\hline GSM1313528 & Hematopoietic stem/progenitor cells & H3K79me2 \\
\hline GSM486702 & Hematopoietic stem/progenitor cells & Input \\
\hline GSM486709 & Hematopoietic stem/progenitor cells & $\mathrm{H} 3 \mathrm{~K} 4 \mathrm{me} 3$ \\
\hline GSM486711 & Hematopoietic stem/progenitor cells & $\mathrm{H} 3 \mathrm{~K} 4 \mathrm{me} 3$ \\
\hline GSM537629 & Hematopoietic stem/progenitor cells & $\mathrm{H} 3 \mathrm{~K} 4 \mathrm{me} 3$ \\
\hline GSM537652 & Hematopoietic stem/progenitor cells & $\mathrm{H} 3 \mathrm{~K} 4 \mathrm{me} 3$ \\
\hline GSM537662 & Hematopoietic stem/progenitor cells & Input \\
\hline GSM621404 & Hematopoietic stem/progenitor cells & $\mathrm{H} 3 \mathrm{~K} 4 \mathrm{me} 3$ \\
\hline GSM621437 & Hematopoietic stem/progenitor cells & $\mathrm{H} 3 \mathrm{~K} 4 \mathrm{me} 3$ \\
\hline GSM621439 & Hematopoietic stem/progenitor cells & $\mathrm{H} 3 \mathrm{~K} 4 \mathrm{me} 3$ \\
\hline GSM621456 & Hematopoietic stem/progenitor cells & Input \\
\hline GSM621665 & Hematopoietic stem/progenitor cells & $\mathrm{H} 3 \mathrm{~K} 4 \mathrm{me} 3$ \\
\hline GSM621667 & Hematopoietic stem/progenitor cells & $\mathrm{H} 3 \mathrm{~K} 4 \mathrm{me} 3$ \\
\hline GSM621689 & Hematopoietic stem/progenitor cells & Input \\
\hline GSM669929 & Hematopoietic stem/progenitor cells & Input \\
\hline GSM669943 & Hematopoietic stem/progenitor cells & $\mathrm{H} 3 \mathrm{~K} 4 \mathrm{me} 3$ \\
\hline GSM669961 & Hematopoietic stem/progenitor cells & Input \\
\hline GSM706846 & Hematopoietic stem/progenitor cells & Input \\
\hline GSM706847 & Hematopoietic stem/progenitor cells & Input \\
\hline GSM772870 & Hematopoietic stem/progenitor cells & $\mathrm{H} 3 \mathrm{~K} 27 \mathrm{ac}$ \\
\hline GSM772885 & Hematopoietic stem/progenitor cells & $\mathrm{H} 3 \mathrm{~K} 27 \mathrm{ac}$ \\
\hline GSM772894 & Hematopoietic stem/progenitor cells & $\mathrm{H} 3 \mathrm{~K} 27 \mathrm{ac}$ \\
\hline GSM772950 & Hematopoietic stem/progenitor cells & Input \\
\hline GSM773041 & Hematopoietic stem/progenitor cells & $\mathrm{H} 3 \mathrm{~K} 4 \mathrm{me} 3$ \\
\hline GSM773044 & Hematopoietic stem/progenitor cells & Input \\
\hline GSM773045 & Hematopoietic stem/progenitor cells & Input \\
\hline GSM773048 & MLL-AF9 leukemia cells & Input \\
\hline GSM1313524 & MLL-AF9 leukemia cells & $\mathrm{H} 3 \mathrm{~K} 4 \mathrm{me} 3$ \\
\hline GSM1313525 & MLL-AF9 leukemia cells & H3K79me2 \\
\hline GSM1313530 & MLL-AF9 leukemia cells & Input \\
\hline GSM2108046 & MLL-AF9 leukemia cells & $\mathrm{H} 3 \mathrm{~K} 27 \mathrm{ac}$ \\
\hline GSM2108047 & MLL-AF9 leukemia cells & $\mathrm{H} 3 \mathrm{~K} 4 \mathrm{me} 3$ \\
\hline GSM2108048 & MLL-AF9 leukemia cells & H3K79me2 \\
\hline GSM2366240 & MLL-AF9 leukemia cells & $\mathrm{H} 3 \mathrm{~K} 4 \mathrm{me} 3$ \\
\hline GSM2366241 & MLL-AF9 leukemia cells & $\mathrm{H} 3 \mathrm{~K} 4 \mathrm{me} 3$ \\
\hline GSM2366243 & MLL-AF9 leukemia cells & $\mathrm{H} 3 \mathrm{~K} 4 \mathrm{me} 3$ \\
\hline GSM2366245 & MLL-AF9 leukemia cells & $\mathrm{H} 3 \mathrm{~K} 4 \mathrm{me} 3$ \\
\hline GSM2366246 & MLL-AF9 leukemia cells & $\mathrm{H} 3 \mathrm{~K} 4 \mathrm{me} 3$ \\
\hline GSM2366247 & MLL-AF9 leukemia cells & $\mathrm{H} 3 \mathrm{~K} 4 \mathrm{me} 3$ \\
\hline GSM2366248 & MLL-AF9 leukemia cells & $\mathrm{H} 3 \mathrm{~K} 4 \mathrm{me} 3$ \\
\hline GSM721210 & MLL-AF9 leukemia cells & H3K79me2 \\
\hline
\end{tabular}

GEO, Gene Expression Omnibus; H3K79me2, histone 3 lysine 79 dimethylation; H3K4me3, histone 3 lysine 4 trimethylation; H3K27ac, histone 3 lysine 27 acetylation; MLL-AF9, mixed lineage leukemia-MLLT3, super elongation complex subunit.

cultured in RPMI-1640 medium (Hyclone; GE Healthcare Life Sciences) supplemented with $20 \%$ fetal bovine serum (Gibco; Thermo Fisher Scientific, Inc.), $100 \mathrm{ng} / \mathrm{ml}$ stem cell factor, $10 \mathrm{ng} / \mathrm{ml}$ interleukin (IL)-3 and $10 \mathrm{ng} / \mathrm{ml}$ IL-6 (R\&D Systems, Inc.). Retrovirus was produced following infection of 293T cells (The Institution of Biochemistry and Cell Biology,
Chinese Academy of Sciences) with retroviral construct pMIG-FLAG-MLL-AF9 (Addgene plasmid 71443; Addgene, Inc.) along with the helper plasmid pCL-ECO (Addgene plasmid 12371; Addgene, Inc.) and the virus supernatants was harvested $48 \mathrm{~h}$ after transfection, filtered through a $0.45 \mu \mathrm{m}$ syringe filter. The lineage negative hematopoietic 
progenitors were infected by spinfection $(657 \mathrm{~g}$ for $2 \mathrm{~h}$ at $32^{\circ} \mathrm{C} ; 4 \mu \mathrm{g} / \mu \mathrm{l}$ polybrene). pMIG-FLAG-MLL-AF9 was a gift from Professor Daisuke Nakada (Addgene plasmid no. 71443; http://n2t.net/addgene:71443; RRID, Addgene_71443) (17). pCL-Eco was a gift from Professor Inder Verma (Addgene plasmid no. 12371; http://n2t.net/addgene:12371; RRID, Addgene_12371) (18). Infection efficiency was shown to be 20-25\% as assessed by GFP expression using flow cytometry (BD FACSVerse; BD Biosciences; Becton, Dickinson and Company). Recipient C57BL/6 mice were transferred from SPF conditions to the RS 2000 biological irradiator ( $\mathrm{Rad}$ Source Technologies, Inc). The X-ray-irradiation lasted $5 \mathrm{~min}$ and $52 \mathrm{sec}$ with a total dose rate of $7.5 \mathrm{~Gy}$. After irradiation, the animals were placed back to their cages under SPF conditions. The infected cells were intravenously injected via the tail vein into X-ray-irradiated recipient C57BL/6 mice $48 \mathrm{~h}$ after infection $\left(10^{6}\right.$ transduced cells per mouse). All recipient mice were housed in a specific pathogen-free environment and fed autoclaved water ( $\mathrm{pH}$ 2.0). Retroviral construct pMIG-FLAG-MLL-AF9 co-expressed MLL-AF9 along with a green fluorescent protein (GFP) reporter gene, thus GFP-positive cells indicated effective MLL-AF9 virus infection. Peripheral blood was collected into Eppendorf tubes from the mouse tail with EDTA anticoagulant, incubated with red blood lysis buffer (Beijing Solarbio Science \& Technology Co., Ltd.) and subjected to flow cytometry analysis. The recipients were evaluated for the percentage of GFP-positive cells in peripheral blood 3 weeks after injection using flow cytometry (BD FACSVerse; BD Biosciences; Becton, Dickinson and Company). The control recipients received the same dose of radiation and were intravenously injected with normal lineage negative hematopoietic progenitors via the tail vein. The percentage of GFP-positive cells increased with time in MLL-AF9 model mice.

Reverse transcription-quantitative polymerase chain reaction $(R T-q P C R)$ analysis. Total RNA samples harvested from the whole bone marrow cells of sacrificed MLL-AF9 mice and WT C57BL/6 mice were extracted using TRIzol reagent (Invitrogen; Thermo Fisher Scientific, Inc.). cDNA was synthesized using Takara PrimeScript RT Master Mix (cat.no. RR036A; Takara Biotechnology Co., Ltd.) according to the manufacturer's protocols. The RT reaction was performed as follows: $15 \mathrm{~min}$ at $37^{\circ} \mathrm{C}$ followed by an incubation at $85^{\circ} \mathrm{C}$ for 5 sec. qPCR was performed with SYBR Premix Ex Taq (cat. no. DRR041A; Takara Biotechnology Co., Ltd.) using an Applied Biosystems 7500 Real-Time PCR system (Thermo Fisher Scientific, Inc.) following manufacturer's protocols. The thermocycling conditions were as follows: Initial denaturation at $95^{\circ} \mathrm{C}$ for $30 \mathrm{sec}$, followed by 40 cycles of $95^{\circ} \mathrm{C}$ for $5 \mathrm{sec}$ and $60^{\circ} \mathrm{C}$ for $30 \mathrm{sec}$. Gene expression was normalized to the levels of mouse GAPDH mRNA. Relative mRNA expression was calculated using the $2^{-\Delta \Delta \mathrm{Cq}}$ method (19). Primer sequences are listed in Table II.

Data analysis and statistics. Kaplan-Meier survival analysis and log-rank test were performed to determine the overall survival of MLL-AF9 mice and WT C57BL/6 mice. Leukemic burden in peripheral blood and splenomegaly between groups were compared using Student's t-test. Statistical analyses
Table II. Primer for reverse transcription-quantitative polymerase chain reaction.

\begin{tabular}{ll}
\hline Gene & \multicolumn{1}{c}{ Sequence } \\
\hline HOXA9 & Sense: 5'-CCCCGACTTCAGTCCTTGC-3' \\
& Antisense: 5'-GATGCACGTAGGGGTGGTG-3' \\
HOXA5 & Sense: 5'-CTCATTTTGCGGTCGCTATCC-3' \\
& Antisense: 5'-ATCCATGCCATTGTAGCCG \\
& TA-3' \\
HOXA10 & Sense: 5'-CCTGCCGCGAACTCCTTTT-3' \\
& Antisense: 5'-GGCGCTTCATTACGCTTGC-3' \\
GNB4 & Sense: 5'-CAGGAGGCTGAACAGCTTCG-3' \\
& Antisense: 5'-GGCCCACGGAGTCCATATTA-3' \\
SESN3 & Sense: 5'-CGGAAGGACAAAAGAATCCGA-3' \\
& Antisense: 5'-GTTCATCCGCCGTATTTGCT-3' \\
PBX3 & Sense: 5'-CGAGGCGCAAGCAAAGAAAC-3' \\
& Antisense: 5'-TGCCAAAAGCATATTGTCCA \\
& GT-3' \\
PLSCR1 & Sense: 5'-GGTATCCCCCTCCGTATCCAC-3' \\
& Antisense: 5'-GCCACCACCTGCATAACCT-3' \\
& Sense: 5'-ATGACTGTGTTCAGGCAGGAA-3' \\
DAPK1 & Antisense: 5'-CCGGTACTTTTCTCACGACAT \\
& TT-3'
\end{tabular}

HOXA, homeobox A; GNB4, G protein subunit $\beta$ 4; SESN3, sestrin 3; PBX3, PBX homeobox 3; PLSCR1, phospholipid scramblase 1; DAPK1, death associated protein kinase 1.

were performed using statistical software $\mathrm{R}$ (version 3.3.3; www.r-project.org/) and survival package. $\mathrm{P}<0.05$ was considered to indicate a statistically significant difference.

\section{Results}

Study overview. The present study identified DEGs from two independent datasets and mapped them to GO and KEGG pathways to identify significantly enriched functional terms. Histone modification changes in MLL-AF9 binding sites were further profiled based on public ChIP-Seq datasets. The potential direct targets of MLL-AF9 were identified by comparing alterations in histone modifications with changes in gene expression. The differential expression of potential direct targets of MLL-AF9 was validated in the public AML project TARGET and by RT-qPCR in an MLL-AF9 mouse model. A flowchart of presenting the study design is shown in Fig. 1.

DEGs in MLL-AF9 AML. To identify DEGs associated with MLL-AF9, two independent datasets (GSE68643 and GSE73457) were analyzed and murine MLL-AF9 leukemia cells were compared with normal hematopoietic cells to detect common genes across these datasets. The analyses revealed that 413 genes were consistently identified as being differentially expressed (adjusted $\mathrm{P}<0.05$ ) with $\geq 2$-fold differential expression between the groups (Fig. 2A). The $\log 2 \mathrm{FC}$ of common DEGs exhibited a high correlation (Pearson's correlation coefficient value $=0.78$ ) between these two independent 


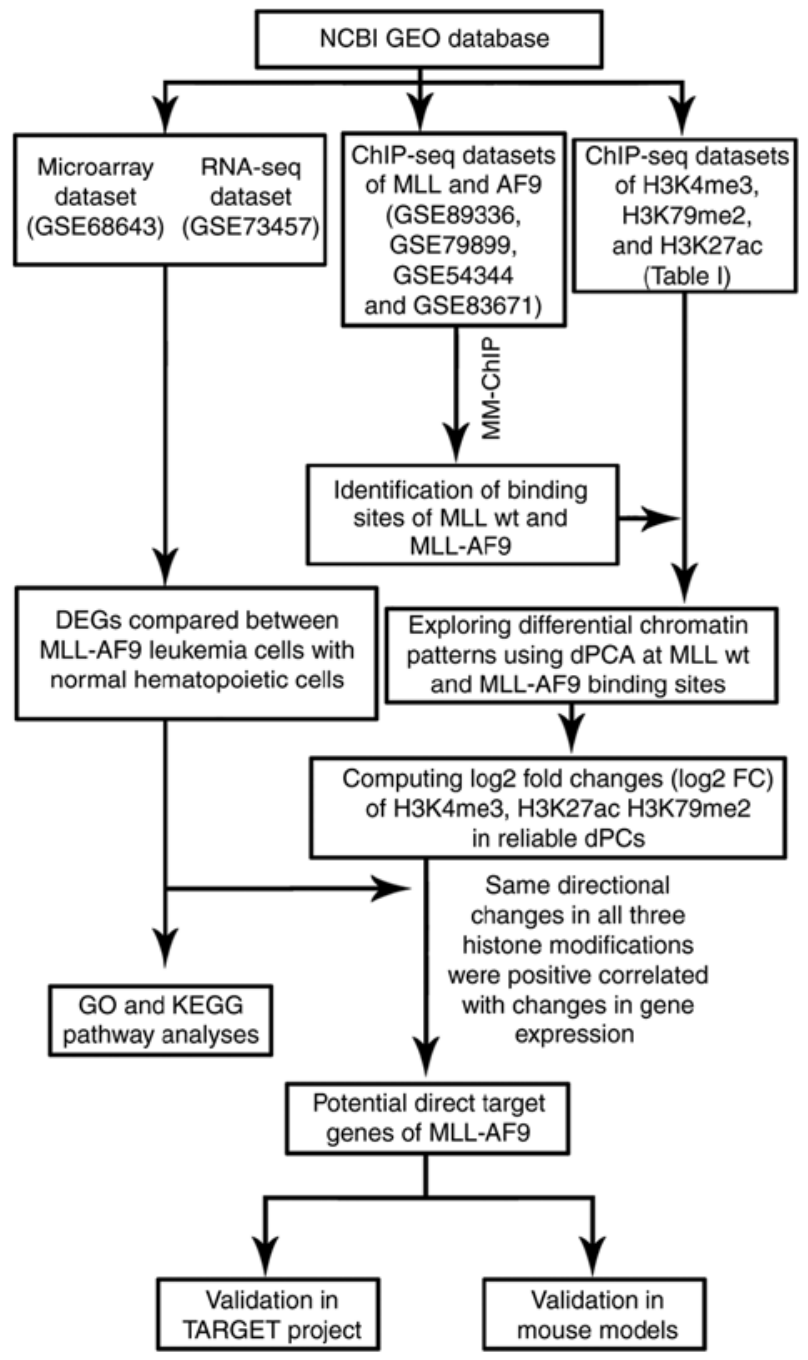

Figure 1. Study schematic. NCBI, National Center for Biotechnology Information; GEO, Gene Expression Omnibus; ChIP, chromatin immunoprecipitation; MLL, mixed lineage leukemia; AF9, MLLT3, super elongation complex subunit; H3K4me3, histone 3 lysine 4 trimethylation; H3K79me2, histone 3 lysine 79 dimethylation; H3K27ac, histone 3 lysine 27 acetylation; wt, wild-type; DEGs, differentially expressed genes; dPCA, differential principal component analysis; dPCs, differential principal components; GO, Gene Ontology; KEGG, Kyoto Encyclopedia of Genes and Genomes; TARGET, the Therapeutically Applicable Research to Generate Effective Treatments project.

experiments. Of these, 57 and 356 genes were upregulated and downregulated, respectively (Fig. 2B). There were various genes previously demonstrated to be associated with MLL-AF9, including the homeobox A (HOXA) cluster genes, Meis homeobox 1, $\beta 3$ integrin and runt related transcription factor 2. These results suggested that the datasets used in the present study were suitable for MLL-AF9 gene analysis.

$G O$ and KEGG functional enrichment analysis of common $D E G s$. The common DEGs were then subjected to enrichment analysis in DAVID. The common DEGs in blood were enriched in biological processes of 'immune system process', 'inflammatory response', 'immune response', 'signal transduction', 'innate immune response', 'adaptive immune response', 'chemotaxis', 'positive regulation of ERK1 and ERK2 cascade', 'positive regulation of $\mathrm{T}$ cell proliferation', 'positive regulation
A

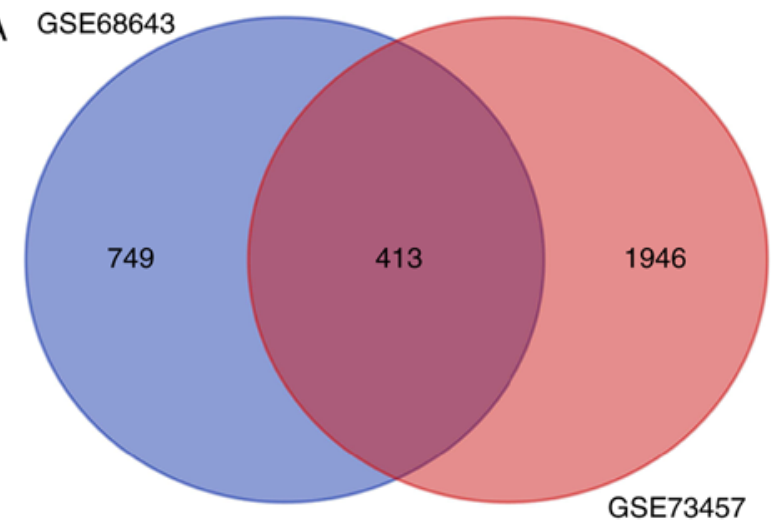

B

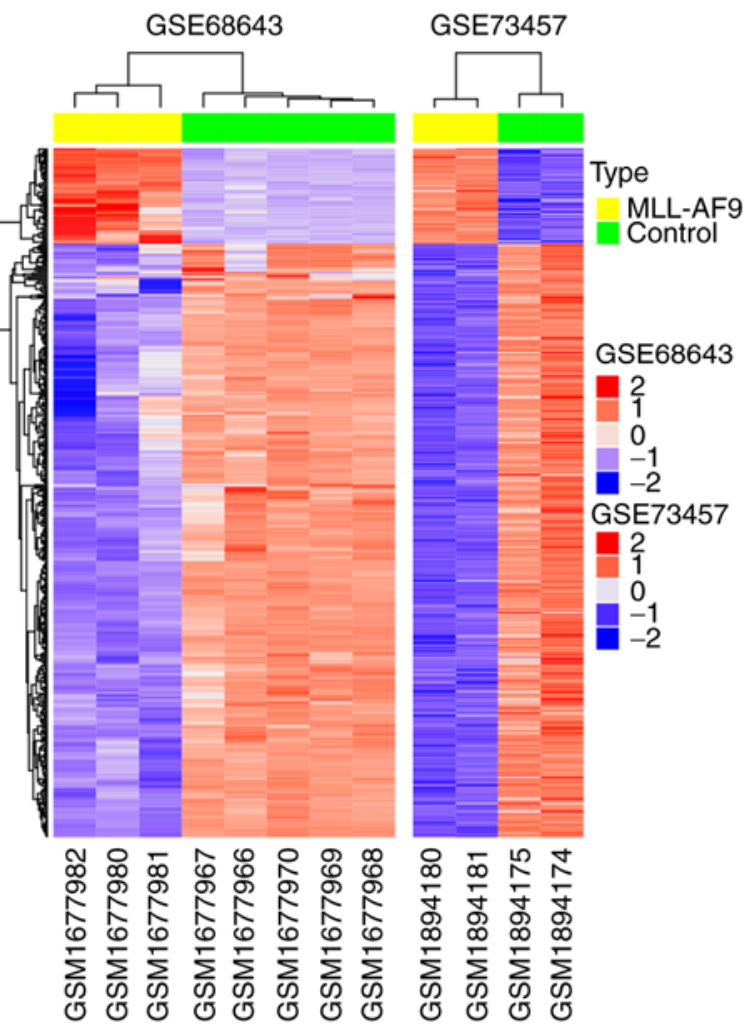

Figure 2. Identification of DEGs in MLL-AF9 acute myeloid leukemia mouse models. (A) Venn diagram of overlapping genes in the analyses result of GSE68643 and GSE73457 datasets. (B) Heatmap of DEGs. DEGs, differentially expressed genes; MLL-AF9, mixed lineage leukemia-MLLT3, super elongation complex subunit.

of cell migration', 'embryonic skeletal system morphogenesis', 'negative regulation of inflammatory response', 'positive regulation of angiogenesis' and 'response to lipopolysaccharide' (Fig. 3A). KEGG enrichment analysis revealed that DEGs were enriched in pathways including the 'nuclear factor- $\mathrm{KB}(\mathrm{NF}-\mathrm{\kappa} \mathrm{B})$ signaling pathway', 'tumor necrosis factor (TNF) signaling pathway', 'cytokine-cytokine receptor interaction', hematopoietic cell lineage', 'sphingolipid signaling pathway', 'T cell receptor signaling pathway', 'transcriptional misregulation in cancer' and 'measles' (Fig. 3B).

Identification of MLL-AF9 and MLL WT binding peaks. Using MM-ChIP for integrative peaks analysis and HOMER software for overlapping peaks. The present study identified 10,464 MLL-AF9 occupied regions and 11,722 WT MLL 
A

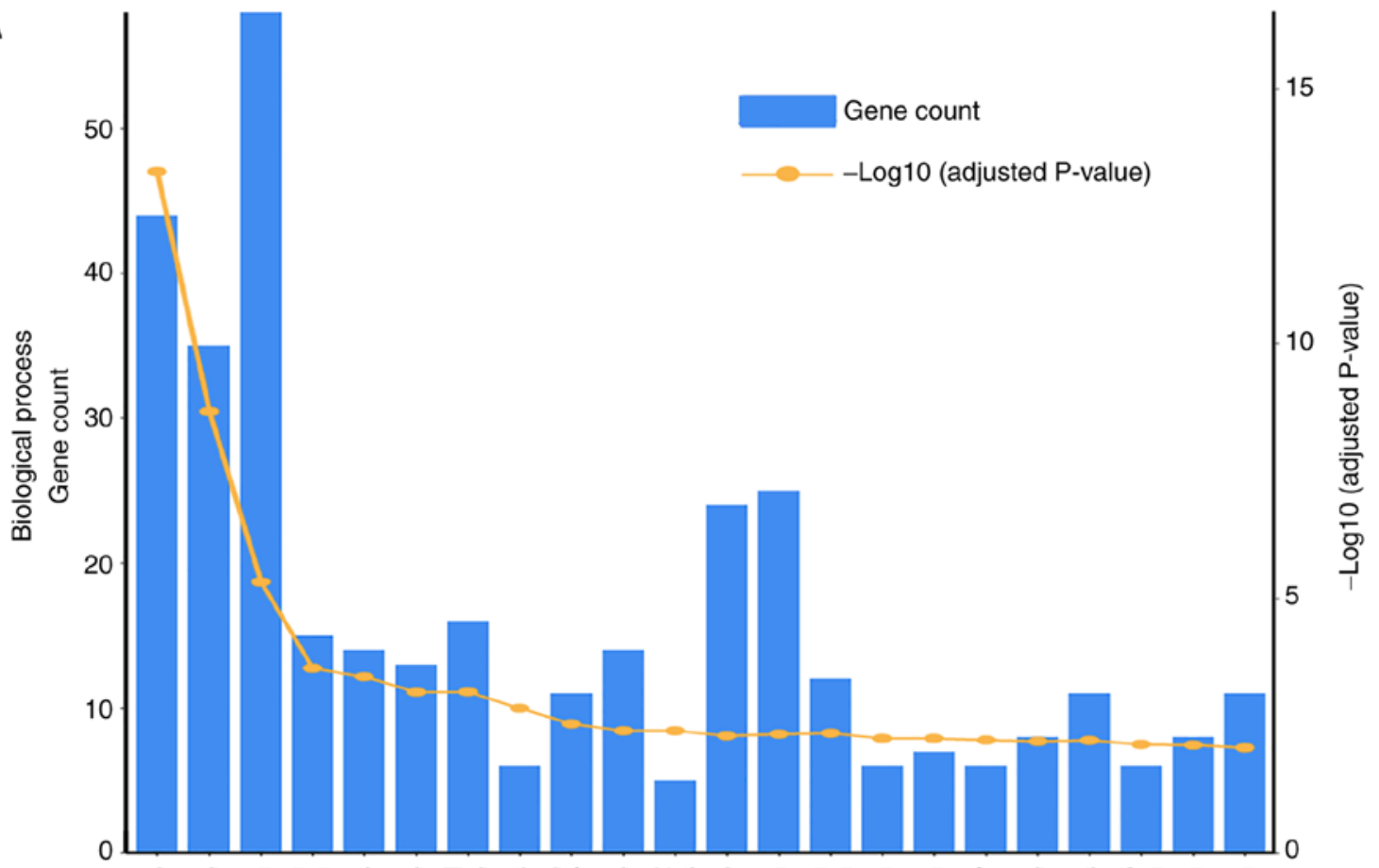

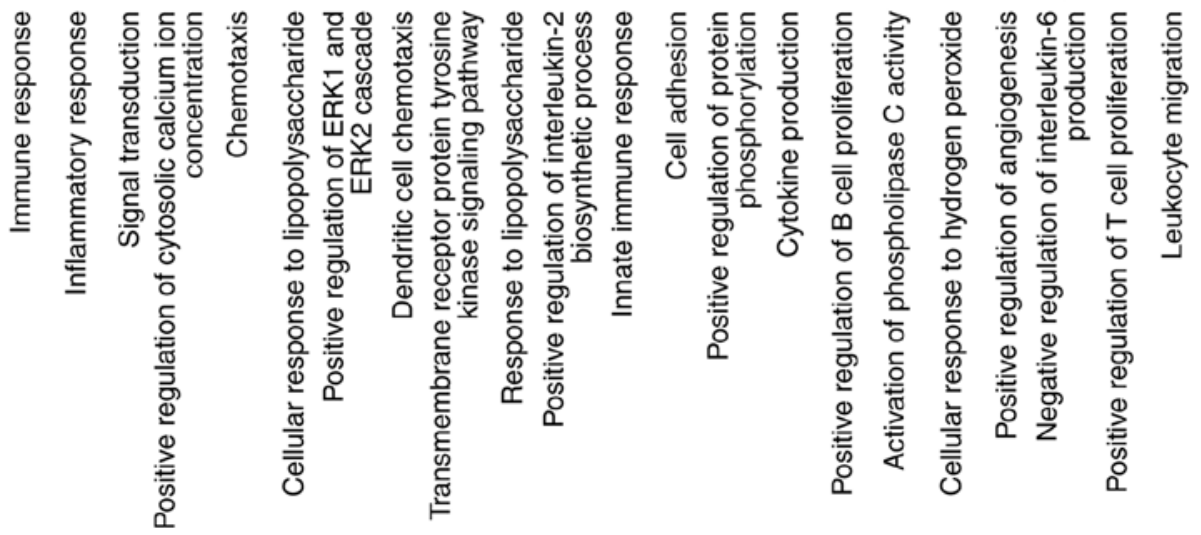

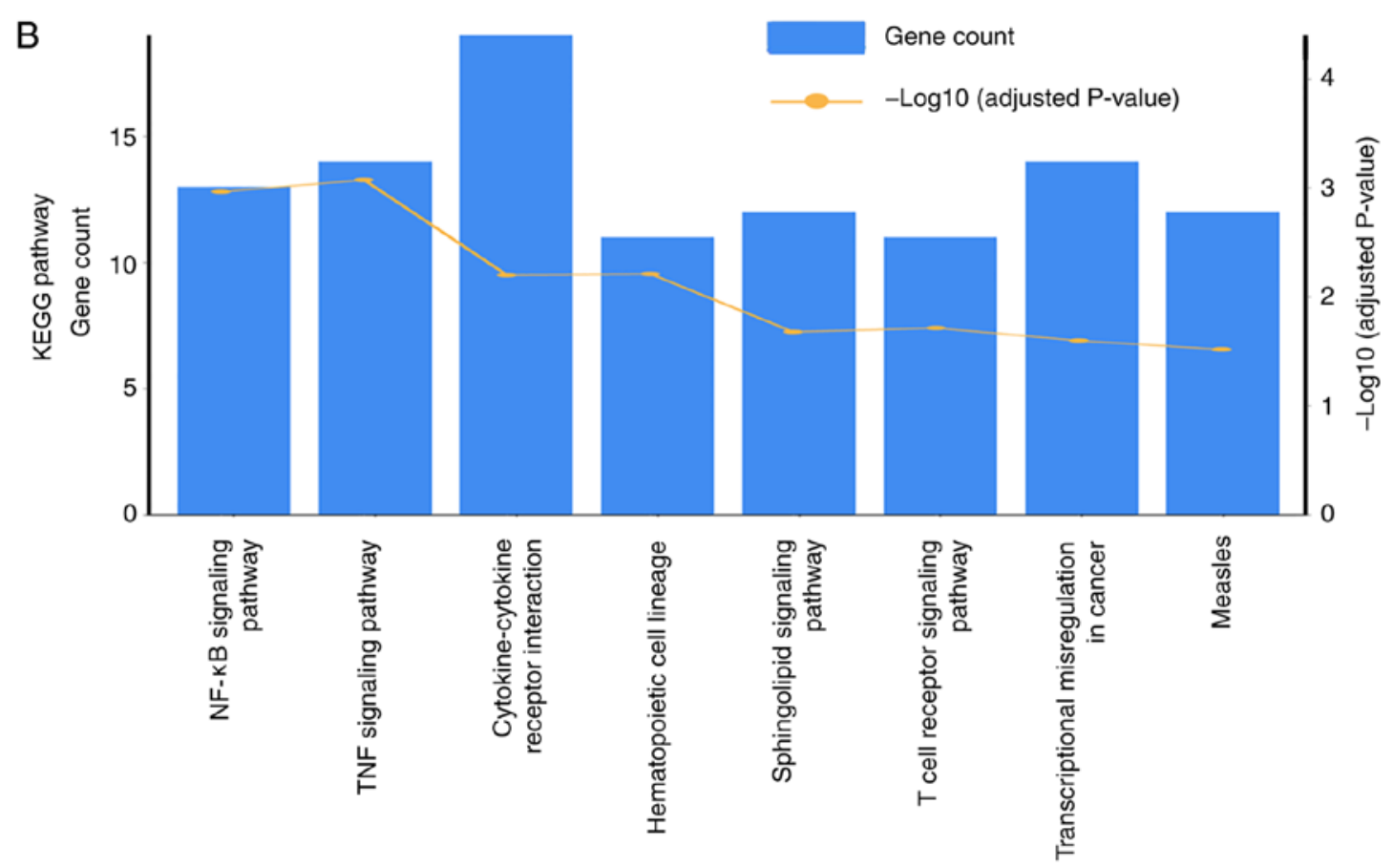

Figure 3. Functional enrichment analyses of common DEGs. (A) Gene Ontology enrichment based on biological process. (B) KEGG enrichment analysis of DEGs. DEGs, differentially expressed genes; ERK, extracellular signal-regulated kinase; KEGG, Kyoto Encyclopedia of Genes and Genomes; NF- $\mathrm{BB}$, nuclear factor- $\mathrm{kB}$; TNF, tumor necrosis factor. 

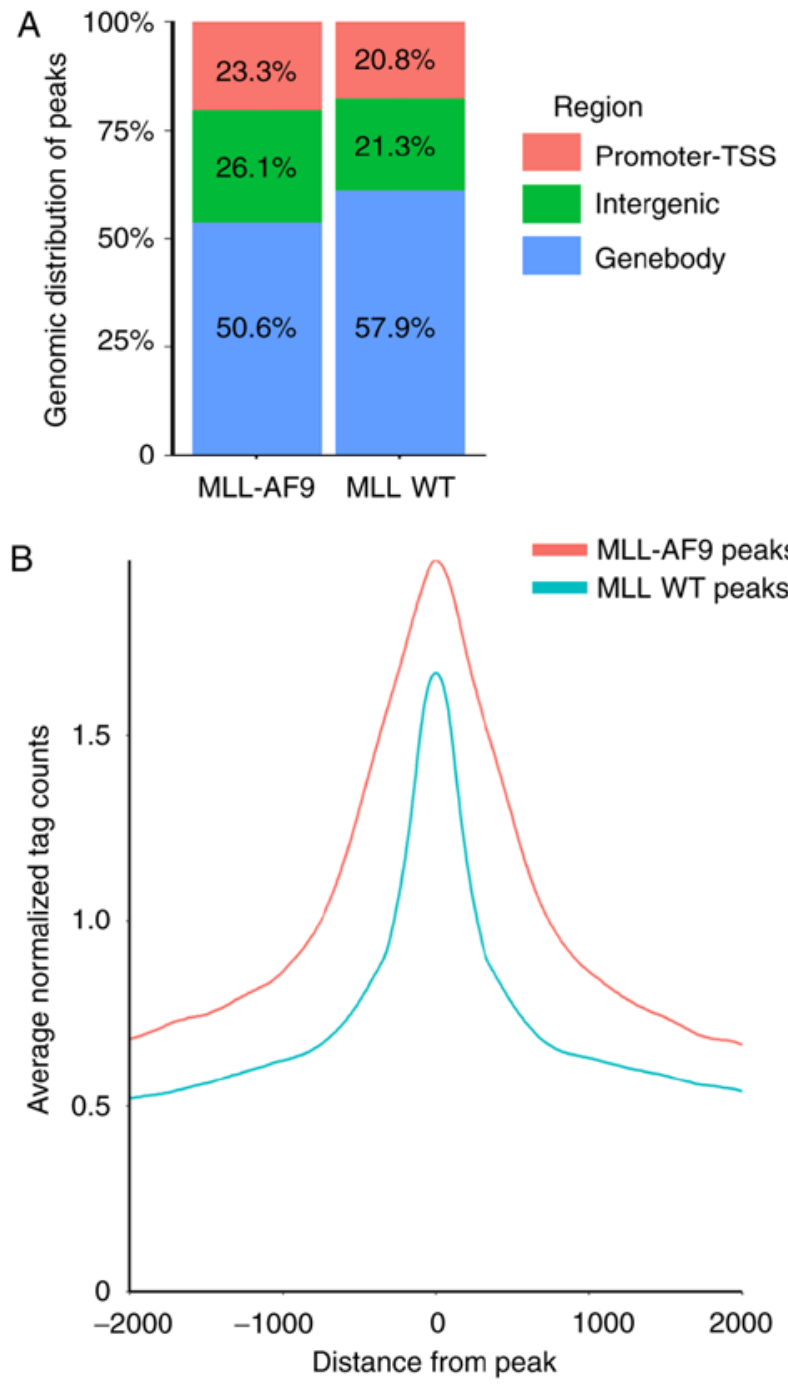

Figure 4. Identification of MLL-AF9 and MLL WT binding sites. (A) Genomic distribution of the MLL-AF9 and MLL WT binding sites. (B) Chromatin immunoprecipitation-seq signal strength of the MLL-AF9 and MLL WT binding sites. TSS, transcriptional start site; MLL, mixed lineage leukemia; AF9, MLLT3, super elongation complex subunit; WT, wild-type.

occupied regions. There was no significant difference in genomic distribution between the MLL-AF9 and MLL WT peaks (Fig. 4A). However, MLL-AF9 peaks exhibited a higher signal than the MLL WT peaks (Fig. 4B).

Identification of differential histone modifications at MLL-AF9 binding sites between MLL-AF9 leukemia cells and normal hematopoietic cells. To systematically investigate the aberrant histone modifications involved in MLL-AF9, ChIP-Seq data of histone modifications in MLL-AF9 leukemia cells and normal hematopoietic progenitor cells were downloaded from NCBI GEO datasets, including H3K4me3, H3K79me2 and H3K27ac. dPCA was applied to explore differential chromatin patterns at the MLL-AF9 peaks and MLL-WT peaks (Table I). The top dPCs in MLL-AF9 exhibited SNR $>5$, while the SNR in dPCs of MLL-WT peaks displayed SNR $<5$ (Fig. 5). Thus, only significant histone modifications differences were observed in MLL-AF9 binding sites. At the 5\% FDR level, dPCA reported 4,166 differential histone modifications sites.

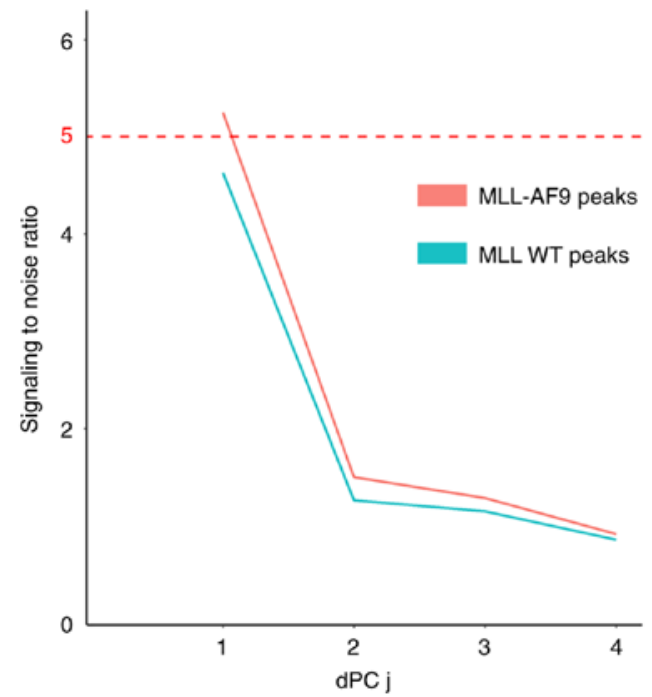

Figure 5. Estimated signal-to-noise ratio for each dPC. MLL, mixed lineage leukemia; AF9, MLLT3, super elongation complex subunit; dPCs, differential principal components; $\mathrm{j}, \mathrm{dPC} 1$.

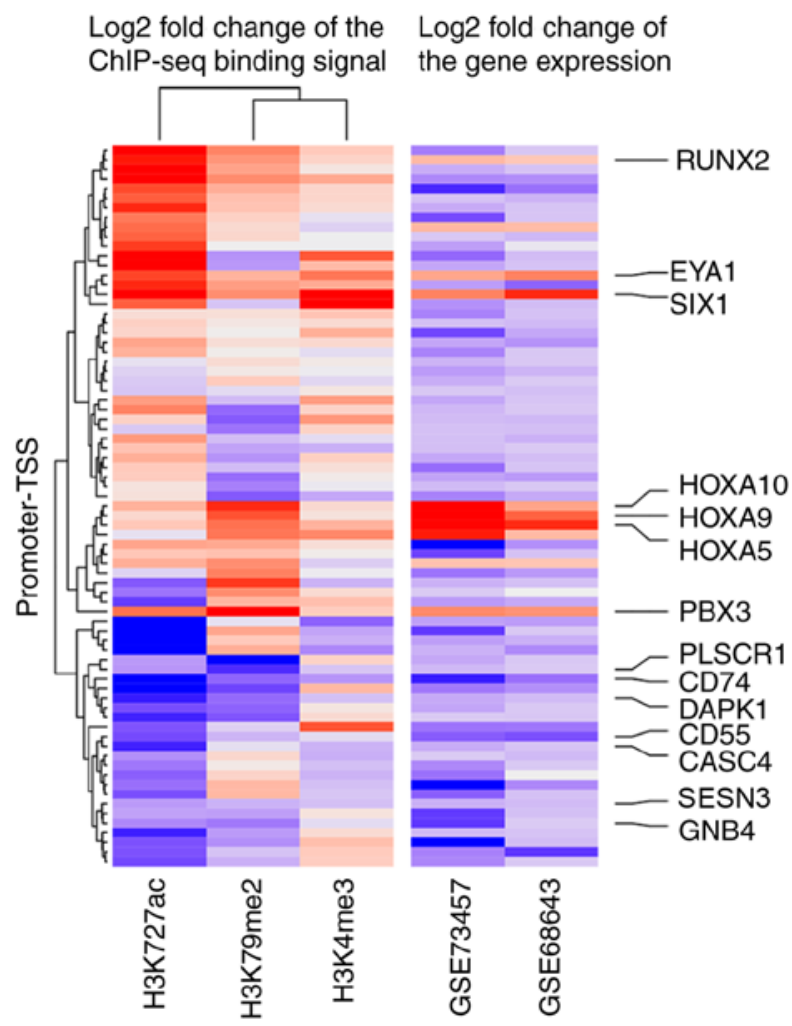

Figure 6. $\log 2$ fold change of DEGs and the $\log 2$ fold change of H3K4me3, $\mathrm{H} 3 \mathrm{~K} 27 \mathrm{ac}$ and $\mathrm{H} 3 \mathrm{~K} 79 \mathrm{me} 2$ binding signals in promoter-TSS regions. TSS, transcriptional start site; RUNX2, runt related transcription factor 2; EYA1, EYA transcriptional coactivator and phosphatase 1; SIX1, SIX homeobox 1; HOXA, homeobox A; PBX3, PBX homeobox 3; PLSCR1, phospholipid scramblase 1; DAPK1, death associated protein kinase 1; CASC4, cancer susceptibility 4; SESN3, sestrin 3; GNB4, G protein subunit $\beta$ 4; H3K27ac, histone 3 lysine 27 acetylation; H3K79me2, histone 3 lysine 79 dimethylation; H3K4me3, histone 3 lysine 4 trimethylation.

Correlation between DEGs and differential chromatin signatures. A total of 390 differential histone modifications sites were associated with 220 MLL-AF9 DEGs, accounting 

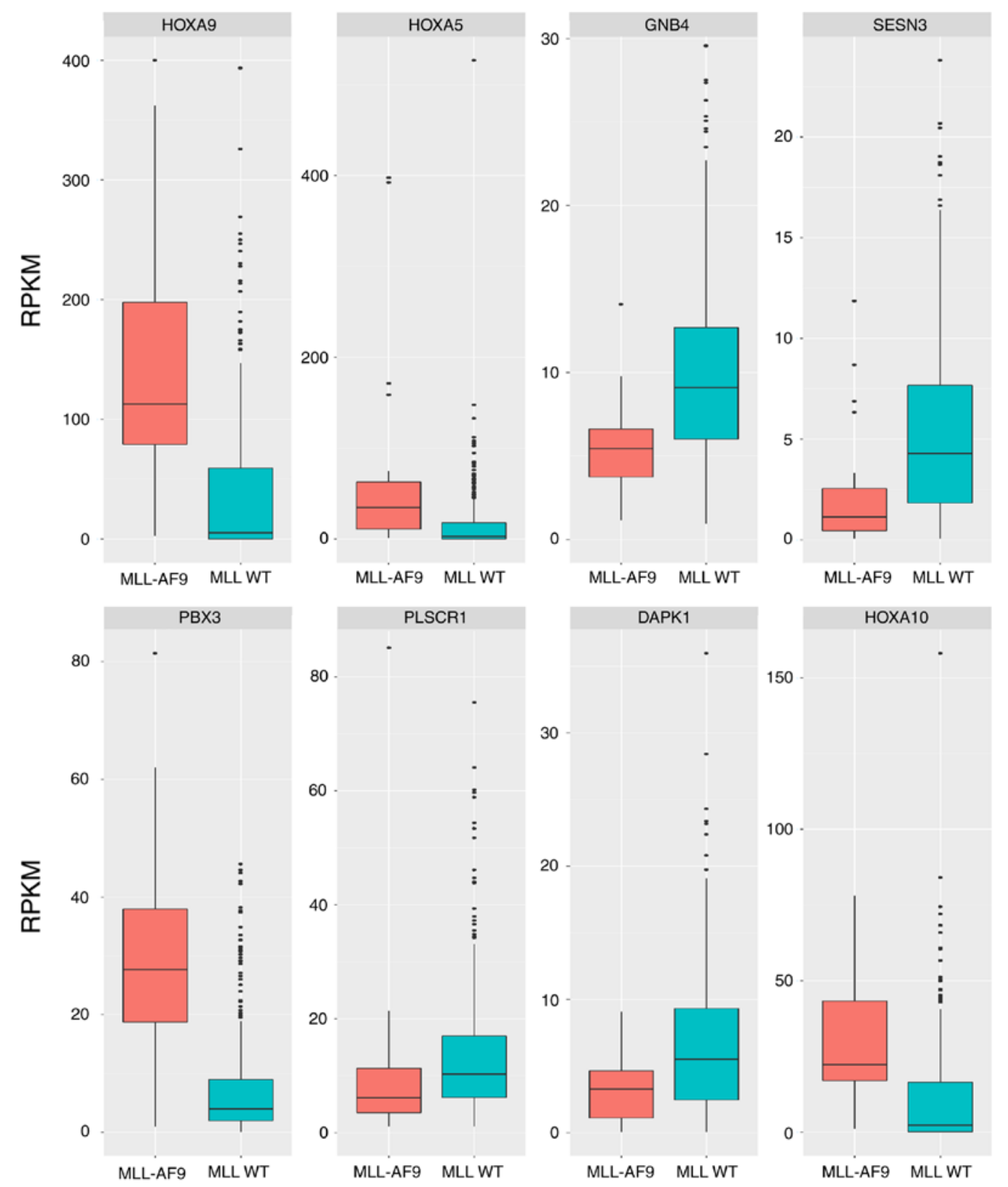

Figure 7. Validation of expression profiles of potential direct target genes of MLL-AF9 in the TCGA-TARGET-GTEx cohort database comparing acute myeloid leukemia samples with MLL-AF9 translocation and normal blood samples. FPKM, fragments per kilobase per million; HOXA, homeobox A; MLL, mixed lineage leukemia; AF9, MLLT3, super elongation complex subunit; GNB4, G protein subunit $\beta$ 4; SESN3, sestrin 3; PBX3, PBX homeobox 3; PLSCR1, phospholipid scramblase 1; DAPK1, death associated protein kinase 1; AML, acute myeloid leukemia; TCGA, The Cancer Genome Atlas; TARGET, Therapeutically Applicable Research to Generate Effective Treatments; GTEx, Genotype-Tissue Expression project.

for $53.3 \%$ of all DEGs. As H3K4me3, H3K27ac and H3K79me2 are known as active transcription activities, the same directional changes in all three histone modifications were associated with an increase in gene expression. As presented in Fig. 6, a specific epigenetic signature was identified and the expression difference of 14 genes was highly associated with the $\log _{2} \mathrm{FC}$ of $\mathrm{H} 3 \mathrm{~K} 4 \mathrm{me} 3, \mathrm{H} 3 \mathrm{~K} 27 \mathrm{ac}$ and $\mathrm{H} 3 \mathrm{~K} 79 \mathrm{me} 2$ binding signals in promoter-transcription start site regions when comparing MLL-AF9 leukemia cells and normal hematopoietic cells. These 14 genes were identified as potential direct targets of MLL-AF9.

Validation of expression profiles of potential direct target genes of MLL-AF9 in the TARGET database. The present study validated the epigenetic-regulated genes by comparing the gene expression profiles of AML samples with MLL-AF9 translocation and normal blood samples. The comparisons indicated that 8 of the 14 epigenetic-regulated genes were confirmed to be differentially expressed in AML patients with MLL-AF9 translocation (Fig. 7).

Generation of MLL-AF9 leukemia models and RT-qPCR analysis. The present study constructed a murine AML model by transducing lineage negative hematopoietic progenitors with a retroviral vector encoding human MLL-AF9 and transplanting the infected cells into $7.5 \mathrm{~Gy} \mathrm{X}$-ray-irradiated C57BL/6 mice via the tail vein (Fig. 8A). The recipient mice developed leukemia with an average life span of $\sim 6$ weeks 
A

Lethal irradiation (7.5 Gy)
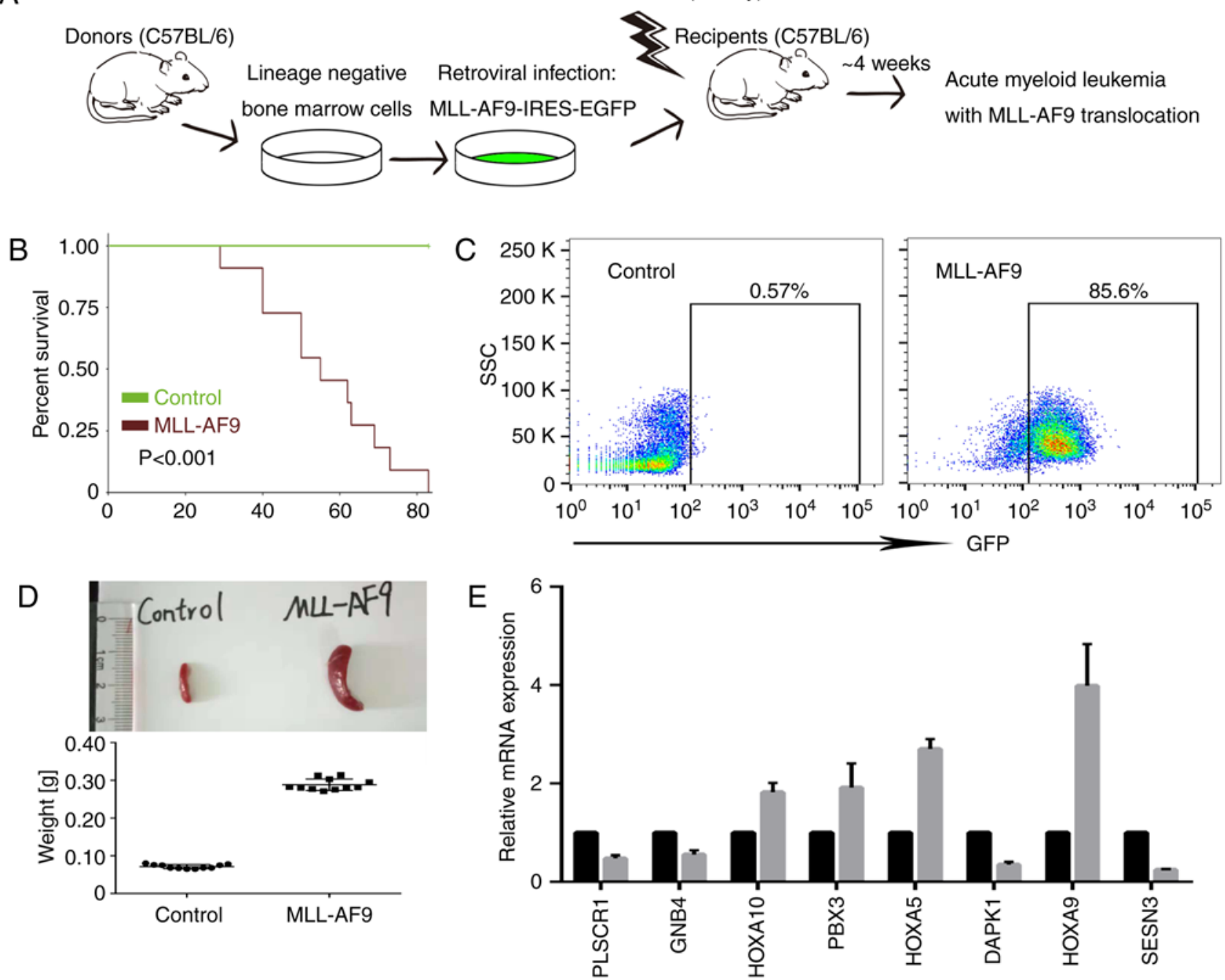

Figure 8. Validation in mouse models. (A) Flow diagram of the animal study. (B) Overall survival of transplanted mice. (C) Representative flow cytometric analysis of GFP-positive cells in the peripheral blood of MLL-AF9 model mice. (D) Sizes and weights of spleens of MLL-AF9 model mice and normal control. (E) The relative mRNA levels of eight potential direct target genes of MLL-AF9. MLL, mixed lineage leukemia; AF9, MLLT3, super elongation complex subunit; SSC, side scatter; GFP, green fluorescent protein; PLSCR1, phospholipid scramblase 1; GNB4, G protein subunit $\beta$ 4; HOXA, homeobox A; PBX3, PBX homeobox 3; DAPK1, death associated protein kinase 1; SESN3, sestrin 3.

(Fig. 8B). The transplanted mice exhibited similar leukemic burden by analysis of GFP+ cells in peripheral blood (Fig. 8C). Leukemic mice were sacrificed and splenomegaly was evaluated when the tumor burden in peripheral blood was $\geq 90 \%$ (Fig. 8D). The expression levels of potential direct target genes of MLL-AF9 were validated by RT-PCR, and the results supported the microarray and RNA-Seq DEG results (GSE68643, GSE73457; Fig. 8E).

\section{Discussion}

AML is a common form of leukemia with an incidence of $\sim 3 / 100,000$ per year $(15,20)$. Patients with MLL-AF9 translocation are defined as a subset of AML with an unfavorable prognosis (21). Epigenetic regulation had been demonstrated to be associated with tumorigenesis in MLL-AF9 leukemia cells. However, previous studies focused on chromatin signatures in MLL-AF9 leukemia cells without comparing them with normal hematopoietic cells $(7,22,23)$. The present study integrated the differential transcriptome and epigenome signatures between MLL-AF9 leukemia cells and normal hematopoietic cells to define the epigenetic-regulated genes in MLL-AF9 leukemia, which would improve understanding of the molecular mechanism in MLL-AF9 leukemia.

Several leukemia-associated biological processes were enriched in the present study, including 'leukocyte migration', 'positive regulation of angiogenesis', 'immune response', 'positive regulation of the ERK1 and ERK2 cascades', 'cell adhesion' and 'transmembrane receptor protein tyrosine kinase signaling pathway'. ITGB3, which is involved in integrin-mediated adhesion, was highly expressed in MLL-AF9 leukemia cells and was determined to be essential for leukemogenesis and chemosensitivity (24). Enriched KEGG terms included, the 'NF- $\mathrm{B}$ signaling pathway', 'TNF signaling pathway', 'cytokine-cytokine receptor interaction', 'hematopoietic cell lineage' and 'transcriptional misregulation in cancer' were closely associated with the initiation and progression of leukemia. The crosstalk mediated by cytokines alters the functional 
characteristics of the mesenchymal stem cells and these effects supported the leukemia cell proliferation and contributed to chemosensitivity (25). The transcription factor NF- $\mathrm{BB}$, which is constitutively activated in the majority of AML cases, controls the expression of genes involved in immune responses and can lead to development of leukemia (26). Furthermore, constitutive NF- $\kappa \mathrm{B}$ activation in the murine MLL-AF9, lysine acetyltransferase 6A-nuclear receptor coactivator 2 or nucleoporin 98-HOXA9 AML models is dependent on autocrine TNF- $\alpha$ signaling (27).

Previous studies have addressed the gene expression changes associated with MLL-AF9 in leukemia cell lines and mouse models. Certain studies focused their research on histone modification in MLL-AF9 leukemia cells $(7,22,28,29)$. The present study differs from the majority of previous reports, as it integrated multiple large-scale histone modification data and compared the chromatin signatures in MLL-AF9 leukemia cells with those in normal hematopoietic cells. The present study identified epigenetic-regulated genes by exploring the association between gene expression and histone modification changes.

Deregulated transcription of HOXA members (HOXA5, HOXA9 and HOXA10) has been identified as a hallmark of MLL rearrangement leukemia (30-32). It was demonstrated that induced expression of PBX homeobox 3 was sufficient for malignant transformation of normal mouse hematopoietic stem/progenitor cells $(33,34)$. Sestrin 3 has been reported to repress ribosomal protein S6 kinase (S6K1) activity by reducing its phosphorylation, which affected the mechanistic target of rapamycin kinase pathway and suppressed leukemic progenitor colony formation in breakpoint cluster region-ABL proto-oncogene 1 , non-receptor tyrosine kinase leukemia cell lines (35). It was demonstrated that inhibition of S6K1 activity impaired self-renewal and improved the survival of mice bearing MLL-AF9 leukemia cells (36). Death associated protein kinase 1 (DAPK1), a tumor suppressor, is involved in the pro-apoptotic activity of TNF- $\alpha$ and interferon- $\gamma$ via the NF- $\kappa B$ signaling pathway (37). Significant suppression of DAPK1 transcription expression levels was observed in primary AML blasts with fms related tyrosine kinase 3-internal tandem duplication or MLL translocation (38). Previous studies reported that phospholipid scramblase 1 has an antagonistic role in leukemia development through the regulation of the cell cycle and cell differentiation $(39,40)$. EYA transcriptional coactivator and phosphatase 1 (EYA1) and SIX homeobox 1 (SIX1) were reported to be associated with leukemogenesis in MLL-MLLT1, super elongation complex subunit AML (28). The present study identified that the average RPKM of EYA1 and SIX1 were 1.34 and 66.04 in mouse bone marrow (GSE73457). However, the average RPKM of EYA1 and SIX1 were 0.06 and 0.01 , respectively, in tumor samples of patients with AML (TARGET project). These two genes may have roles in leukemogenesis in a mouse model, but were expressed at low levels in human hematopoietic cells.

Limitations of the present study must be acknowledged. Additional experiment validation, such as ChIP, is required in future studies. The transcriptomic and epigenetic data analyses revealed that a small subset of DEGs are epigenetic-regulated and these genes may be critical in the leukemogenesis of AML with MLL-AF9 translocation. The results of the present study have provided further insight into the association of chromatin signatures with gene expression. However, further studies are warranted to validate these findings.

\section{Acknowledgements}

The authors would like to thank Professor Daisuke Nakada (Department of Molecular and Human Genetics, Baylor College of Medicine) and Professor Inder Verma (Laboratory of Genetics, The Salk Institute) for providing pMIG-FLAG-MLL-AF9 and pCL-Eco plasmids.

\section{Funding}

The present study was supported by the Ministry of Science and Technology of China (grant no 2016YEE0107200), the National Natural Science Foundation of China (grant no. 81400111) and the Science and Technology Commission of Shanghai Municipality (grant nos. 15411968900 and 15XD1503300).

\section{Availability of data and materials}

The datasets used and/or analyzed during the current study are available from the corresponding authors on reasonable request.

\section{Authors' contributions}

WZ and AL designed the study. FW and ZL performed most of the statistical analyses and drafted the initial manuscript. GW, XT, JZ, WY, LD, JL, JX and ZF contributed to the study design, collected datasets, performed data analysis and revised the paper. The final version of the manuscript was read and approved by all authors.

\section{Ethics approval and consent to participate}

All the animal experiments were performed in accordance with institutional guidelines for Animal Care at Tongji University School of Medicine and received ethical approval from The Animal Ethics Committee of Tongji University (approval no. TJLAC-018-027).

\section{Patient consent for publication}

Not applicable.

\section{Competing interests}

The authors declare that they have no competing interests.

\section{References}

1. Huret JL, Dessen P and Bernheim A: An atlas of chromosomes in hematological malignancies. Example: 11q23 and MLL partners. Leukemia 15: 987-989, 2001.

2. Biondi A, Cimino G, Pieters R and Pui CH: Biological and therapeutic aspects of infant leukemia. Blood 96: 24-33, 2000.

3. Sabattini E, Bacci F, Sagramoso C and Pileri SA: WHO classification of tumours of haematopoietic and lymphoid tissues in 2008: An overview. Pathologica 102: 83-87, 2010. 
4. Marschalek R: Mixed lineage leukemia: Roles in human malignancies and potential therapy. FEBS J 277: 1822-1831, 2010.

5. Andersson AK, Ma J, Wang J, Chen X, Gedman AL, Dang J, Nakitandwe J, Holmfeldt L, Parker M, Easton J, et al: The landscape of somatic mutations in infant MLL-rearranged acute lymphoblastic leukemias. Nat Genet 47: 330-337, 2015.

6. Biswas D, Milne TA, Basrur V, Kim J, Elenitoba-Johnson KS, Allis CD and Roeder RG: Function of leukemogenic mixed lineage leukemia 1 (MLL) fusion proteins through distinct partner protein complexes. Proc Natl Acad Sci USA 108: 15751-15756, 2011.

7. Prange KHM, Mandoli A, Kuznetsova T, Wang SY, Sotoca AM Marneth AE, van der Reijden BA, Stunnenberg HG and Martens JHA: MLL-AF9 and MLL-AF4 oncofusion proteins bind a distinct enhancer repertoire and target the RUNX1 program in 11q23 acute myeloid leukemia. Oncogene 36 3346-3356, 2017

8. Ji H, Li X, Wang QF and Ning Y: Differential principal component analysis of ChIP-seq. Proc Natl Acad Sci USA 110 6789-6794, 2013.

9. Kim D, Langmead B and Salzberg SL: HISAT: A fast spliced aligner with low memory requirements. Nat Methods 12: 357-360, 2015.

10. Anders S, Pyl PT and Huber W: HTSeq--a python framework to work with high-throughput sequencing data. Bioinformatics 31 166-169, 2015

11. Trapnell C, Roberts A, Goff L, Pertea G, Kim D, Kelley DR Pimentel H, Salzberg SL, Rinn JL and Pachter L: Differential gene and transcript expression analysis of RNA-seq experiments with tophat and cufflinks. Nat Protoc 7: 562-578, 2012.

12. Love MI, Huber W and Anders S: Moderated estimation of fold change and dispersion for RNA-seq data with DESeq2. Genome Biol 15: 550, 2014

13. Dennis G Jr, Sherman BT, Hosack DA, Yang J, Gao W, Lane HC and Lempicki RA: DAVID: Database for annotation, visualization, and integrated discovery. Genome Biol 4: P3, 2003.

14. Chen Y, Meyer CA, Liu T, Li W, Liu JS and Liu XS: MM-ChIP enables integrative analysis of cross-platform and betweenlaboratory ChIP-chip or ChIP-seq data.Genome Biol 12:R11,2011

15. Heinz S, Benner C, Spann N, Bertolino E, Lin YC, Laslo P, Cheng JX, Murre C, Singh H and Glass CK: Simple combinations of lineage-determining transcription factors prime cis-regulatory elements required for macrophage and B cell identities. Mol Cell 38: 576-589, 2010.

16. Somervaille TC and Cleary ML: Identification and characterization of leukemia stem cells in murine MLL-AF9 acute myeloid leukemia. Cancer Cell 10: 257-268, 2006.

17. Saito Y, Chapple RH, Lin A, Kitano A and Nakada D: AMPK protects leukemia-initiating cells in myeloid leukemias from metabolic stress in the bone marrow. Cell Stem Cell 17: 585-596, 2015.

18. Naviaux RK, Costanzi E, Haas M and Verma IM: The pCL vector system: Rapid production of helper-free, high-titer, recombinant retroviruses. J Virol 70: 5701-5705, 1996.

19. Livak KJ and Schmittgen TD: Analysis of relative gene expression data using real-time quantitative PCR and the 2(-Delta Delta C(T)) method. Methods 25: 402-408, 2001.

20. Dohner H, Weisdorf DJ and Bloomfield CD: Acute myeloid leukemia. N Engl J Med 373: 1136-1152, 2015.

21. Muntean AG and Hess JL: The pathogenesis of mixed-lineage leukemia. Ann Rev Pathol 7: 283-301, 2012.

22. Bernt KM, Zhu N, Sinha AU, Vempati S, Faber J, Krivtsov AV, Feng Z, Punt N, Daigle A, Bullinger L, et al: MLL-rearranged leukemia is dependent on aberrant H3K79 methylation by DOT1L. Cancer Cell 20: 66-78, 2011.

23. Marneth AE, Prange KHM, Al Hinai ASA, Bergevoet SM, Tesi N, Janssen-Megens EM, Kim B, Sharifi N, Yaspo ML, Kuster J, et al: C-terminal BRE overexpression in 11q23rearranged and $\mathrm{t}(8 ; 16)$ acute myeloid leukemia is caused by intragenic transcription initiation. Leukemia 32: 828-836, 2018.

24. Miller PG, Al-Shahrour F, Hartwell KA, Chu LP, Järås M, Puram RV, Puissant A, Callahan KP, Ashton J, McConkey ME, et al: In vivo RNAi screening identifies a leukemia-specific dependence on integrin beta 3 signaling. Cancer Cell 24: 45-58, 2013.
25. Reikvam H, Brenner AK, Hagen KM, Liseth K, Skrede S, Hatfield KJ and Bruserud $\varnothing$ : The cytokine-mediated crosstalk between primary human acute myeloid cells and mesenchymal stem cells alters the local cytokine network and the global gene expression profile of the mesenchymal cells. Stem Cell Res 15: 530-541, 2015.

26. Cilloni D, Martinelli G, Messa F, Baccarani M and Saglio G: Nuclear factor $\mathrm{kB}$ as a target for new drug development in myeloid malignancies. Haematologica 92: 1224-1229, 2007.

27. Kagoya Y, Yoshimi A, Kataoka K, Nakagawa M, Kumano K, Arai S, Kobayashi H, Saito T, Iwakura Y and Kurokawa M: Positive feedback between NF- $\kappa$ B and TNF- $\alpha$ promotes leukemia-initiating cell capacity. J Clin Invest 124: 528-542, 2014.

28. Wang QF, Wu G, Mi S, He F, Wu J, Dong J, Luo RT, Mattison R, Kaberlein JJ, Prabhakar S, et al: MLL fusion proteins preferentially regulate a subset of wild-type MLL target genes in the leukemic genome. Blood 117: 6895-6905, 2011.

29. Chen L, Sun Y, Wang J, Jiang H and Muntean AG: Differential regulation of the c-Myc/Lin28 axis discriminates subclasses of rearranged MLL leukemia. Oncotarget 7: 25208-25223, 2016

30. Armstrong SA, Staunton JE, Silverman LB, Pieters R, den Boer ML, Minden MD, Sallan SE, Lander ES, Golub TR and Korsmeyer SJ: MLL translocations specify a distinct gene expression profile that distinguishes a unique leukemia. Nat Genet 30: 41-47, 2002

31. Milne TA, Martin ME, Brock HW, Slany RK and Hess JL: Leukemogenic MLL fusion proteins bind across a broad region of the Hox a9 locus, promoting transcription and multiple histone modifications. Cancer Res 65: 11367-11374, 2005.

32. Kawagoe H,Humphries RK, Blair A, Sutherland HJ and Hogge DE: Expression of HOX genes, HOX cofactors, and MLL in phenotypically and functionally defined subpopulations of leukemic and normal human hematopoietic cells. Leukemia 13: 687-698, 1999.

33. Li Z, Chen P, Su R, Hu C, Li Y, Elkahloun AG, Zuo Z, Gurbuxani S, Arnovitz S, Weng H, et al: PBX3 and MEIS1 cooperate in hematopoietic cells to drive acute myeloid leukemias characterized by a core transcriptome of the MLL-Rearranged disease. Cancer Res 76: 619-629, 2016.

34. Guo H, Chu Y, Wang L, Chen X, Chen Y, Cheng H, Zhang L, Zhou Y, Yang FC, Cheng T, et al: PBX3 is essential for leukemia stem cell maintenance in MLL-rearranged leukemia. Int J Cancer 141: 324-335, 2017.

35. Vakana E, Arslan AD, Szilard A, Altman JK and Platanias LC: Regulatory effects of sestrin 3 (SESN3) in BCR-ABL expressing cells. PLoS One 8: e78780, 2013

36. Ghosh J, Kobayashi M, Ramdas B, Chatterjee A, Ma P, Mali RS, Carlesso N, Liu Y, Plas DR, Chan RJ and Kapur R: S6K1 regulates hematopoietic stem cell self-renewal and leukemia maintenance. J Clin Invest 126: 2621-2625, 2016.

37. Yoo HJ, Byun HJ, Kim BR, Lee KH, Park SY and Rho SB: DAPk1 inhibits NF- $\kappa$ B activation through TNF- $\alpha$ and INF- $\gamma$-induced apoptosis. Cell Signal 24: 1471-1477, 2012.

38. Shanmugam R, Gade P, Wilson-Weekes A, Sayar H, Suvannasankha A, Goswami C, Li L, Gupta S, Cardoso AA, Baghdadi TA, et al: A noncanonical Flt3ITD/NF- $\mathrm{B}$ signaling pathway represses DAPK1 in acute myeloid leukemia. Clin Cancer Res 18: 360-369, 2012.

39. Chen Y, Hui H, Yang H, Zhao K, Qin Y, Gu C, Wang X, Lu N and Guo Q: Wogonoside induces cell cycle arrest and differentiation by affecting expression and subcellular localization of PLSCR1 in AML cells. Blood 121: 3682-3691, 2013.

40. Huang Y, Zhao Q, Zhou CX, Gu ZM, Li D, Xu HZ, Wiedmer T, Sims PJ, Zhao KW and Chen GQ: Antileukemic roles of human phospholipid scramblase 1 gene, evidence from inducible PLSCR1-expressing leukemic cells. Oncogene 25: 6618-6627, 2006.

This work is licensed under a Creative Commons Attribution-NonCommercial-NoDerivatives 4.0 International (CC BY-NC-ND 4.0) License. 